\title{
Analysis of 24 cases of polycystic ovary syndrome after failed controlled ovarian hyperstimulation
}

\begin{abstract}
Some fertility patients with polycystic ovary syndrome (PCOS) undergoing controlled ovarian hyperstimulation $(\mathrm{COH})$ experience ceased follicular growth during $\mathrm{COH}$ and fail to superovulate. In this article, 24 such PCOS patients resumed $\mathrm{COH}$ 15-20 days after previous $\mathrm{COH}$ treatment was stopped while patients were under ovarian downregulation. Seventeen cases achieved oocyte retrieval, of which 14 underwent embryo transfer and 12 resulted in clinical pregnancy. The remaining seven cases saw ceased follicular growth at $13 \mathrm{~mm}$. This provides a rescue protocol for failed $\mathrm{COH}$ patients with PCOS.
\end{abstract}

KEYWORDS: PCOS, COH, IVF-ET

\section{Materials and methods \\ Patient inclusion criteria}

Included were a total of 24 patients with polycystic ovary syndrome (PCOS) who underwent in vitro fertilisation-embryo transfer (IVF-ET) treatments at the Reproductive Centre of Women and Children's Hospital, Hunan, China from August 2012 to May 2014. Of these, 13 patients were complicated with pelvic inflammatory disease (PID). The patients were aged between 24 and 40 years with mean age $35.5 \pm 5.1$ and body mass index (BMI) between 15 and 35 with mean BMI 23.5 \pm 5 .09. Patients with BMI more than 25 were required to reduce their body weight more than $5 \mathrm{~kg}$ to be included in the study.

\section{PCOS and PID diagnostic criteria}

The PCOS diagnostic criteria are according to the standard developed by European Society for Human Reproduction (ESHR) and the American Society for Reproductive Medicine (ASRM) revised in 2003 at the Rotterdam PCOS consensus. ${ }^{1}$ The PID diagnostic criteria refer to the standards for diagnosis and treatment of PID made by the infectious diseases group of obstetrics and gynaecology, Chinese Medical Association. ${ }^{2}$ All patients had a history of more than 2 years of infertility, and showed no signs of hydrosalpinx, ovarian cyst and adenomyosis/ endometriosis under vaginal ultrasonography. Prior to their controlled ovarian hyperstimulation $(\mathrm{COH}), 13$ patients had had treatment with clomiphene citrate or letrozol.

\section{Method of treatment}

After being treated with ethinylestradiol and acetate tablets (Bayer) for one to two menstrual cycles, patients were downregulated with 1.1 to $1.4 \mathrm{mg}$ of leuprolide acetate (Lizhu) at midluteal phase for 10 to 14 days, followed by $\mathrm{COH}$ when patients' follicle stimulating hormone to luteinising hormone (FSH/ LH) measured between 1-5 IU/L, oestradiol (E2) <150 pmol/L and progesterone $<2 \mathrm{nmol} / \mathrm{L}$. Gonadotropins for $\mathrm{COH}$ were urine-derived follicle stimulating hormone (FSH; Lizhu) or human menopausal gonadotropins (HMG; Lizhu). The choice of using FSH and/or HMG depended on the level of patient luteinizing hormone (LH). Patients resumed $\mathrm{COH}$ after 15 to 20 days of failed follicle development. The criterion for resuming $\mathrm{COH}$ was that the ovaries were under downregulation which meant that antral follicles on both sides were under $5 \mathrm{~mm}$ after ceased growth follicle had been discharged or had atresia; serum FSH/LH values were less than 5 IU/L, E2 less than $150 \mathrm{pmol} / \mathrm{L}$ and progesterone less than $2 \mathrm{nmol} / \mathrm{L}$; and endometrium was less than $5 \mathrm{~mm}$ in thickness. Twenty-four patients who met criteria described above were selected to resume $\mathrm{COH}$. Of these, seven patients ceased follicle growth again around $13 \mathrm{~mm}$ with declined oestradiol and $\mathrm{COH}$ was cancelled without in vitro maturation (IVM). Seventeen patients with resumed $\mathrm{COH}$ met the criteria for egg retrieval when the dominant follicle was larger than $17 \mathrm{~mm}$ and the oestradiol value was climbing. The total and daily dose of gonadotropin ( $\mathrm{Gn}$ ) for patients in both $\mathrm{COH}$ s was recorded with mean and standard deviation.

\section{Statistical analysis}

The data were analysed using SPSS17.0 software with mean \pm standard deviation, and quantitative data between the two groups with the independent-samples t-test and counts data were analysed with the nonparametric $\chi^{2}$ test.

\section{Results}

\section{Statistical results of E2 and dominant follicles in both $\mathrm{COHs}$}

Twenty-four patients with PCOS had ceased follicular development during their IVF-ET treatment using the long protocol. If we observed that follicles stopped growing and E2 was declining, ovary stimulation was discontinued $2-4$ days after. All patients had dominant follicles less than $16 \mathrm{~mm}$ with mean size $10.56 \pm 2.744 \mathrm{~mm}$ and mean E2 level $785.2 \pm 957.3 \mathrm{pmol} / \mathrm{L}$ when the day of stimulation stopped. After resuming $\mathrm{COH}, 17$ patients successfully developed mature follicles with mean E2 8,747 $\pm 5,111.7$ on the day of HCG trigger. The remaining seven cases showed ceased follicular growth again with mean E2 level $739.3 \pm 789.9$ on the day that medication was discontinued (see Table 1). The different E2 levels between the 17 patients with mature follicles during the second $\mathrm{COH}$ and the 24 patients during the first $\mathrm{COH}$ were statistically significant $(p<0.05)$, but there were no differences in $\mathrm{E} 2$ levels between the seven patients with ceased follicle growth during the second $\mathrm{COH}$ and the 24 patients during the first $\mathrm{COH}(p>0.05)$. 
Table 1. Statistical analysis of the oestradiol and the dominant follicle during both controlled ovarian

hyperstimulations

\begin{tabular}{|c|c|c|c|c|c|c|}
\hline & \multicolumn{2}{|c|}{24 cases in the first $\mathrm{COH}$} & \multicolumn{2}{|c|}{$\begin{array}{l}17 \text { cases were successful in } \\
\text { second } \mathrm{COH}^{a}\end{array}$} & \multicolumn{2}{|c|}{7 cases failed in second $\mathrm{COH}^{\mathrm{b}}$} \\
\hline & E2 (pmol/L) & F-measure $(\mathrm{mm})$ & E2 (pmol/L) & F-measure $(\mathrm{mm})$ & E2 (pmol/L) & F-measure $(\mathrm{mm})$ \\
\hline 4 days prior & $2,103.5 \pm 1941.3$ & $11.2 \pm 3.0$ & $5,623 \pm 4,455$ & $16.2 \pm 3.7$ & $1,879.9 \pm 1,673.2$ & $12.0 \pm 3.1$ \\
\hline 2 days prior & $1,286.3 \pm 1452.3$ & $11.1 \pm 2.7$ & $7,034 \pm 5,021$ & $17.5 \pm 2.8$ & $1,309.1 \pm 1,098.4$ & $12.2 \pm 1.9$ \\
\hline Drug discontinued day & $510.6 \pm 674.8$ & $10.6 \pm 2.7$ & $8,747 \pm 5,111$ & $19.2 \pm 3.2$ & $639.3 \pm 589.9$ & $11.7 \pm 3.0$ \\
\hline $\begin{array}{l}E 2=\text { oestradiol } ; \mathrm{COH}=\text { contrc } \\
a=E 2 \text { for } 17 \text { cases were succ } \\
b=E 2 \text { for seven cases failed }\end{array}$ & $\begin{array}{l}\text { d ovarian hyperstin } \\
\text { sful in second } \mathrm{COH} \\
\text { ain in the second } \mathrm{CC}\end{array}$ & $\begin{array}{l}\text { ulation; } F \text {-measure }=f \\
\text { SE2 for } 24 \text { cases with } \\
\text { H vs E2 for } 24 \text { cases d }\end{array}$ & $\begin{array}{l}\text { fe size. } \\
\text { first } \mathrm{COH}, \mathrm{p}<0.05 \\
\text { g the first } \mathrm{COH}, \mathrm{p}\end{array}$ & & & \\
\hline
\end{tabular}

Total and daily dose of $\mathrm{Gn}$, days of stimulation in both $\mathrm{COHs}$

After 15-20 days of discontinuing medication for stimulation, 24 patients resumed their $\mathrm{COH}$ when they met the criteria for ovary under downregulation. Of these, nine patients received gonadotropin-releasing hormone antagonist (Cetrotide, MerckSerono) to suppress elevated LH to 1-5 IU/L at the late stage of second $\mathrm{COH}$. The total and daily doses of $\mathrm{Gn}$ used in both $\mathrm{COH}$ were recorded (see Table 2). Table 2 shows that the total dose of $\mathrm{Gn}$ for the first $\mathrm{COH}$ and the second $\mathrm{COH}$ were 2,004 \pm 393.5 and $2,628 \pm 970.7$ respectively; the daily dose of $\mathrm{Gn}$ for the first $\mathrm{COH}$ and second $\mathrm{COH}$ were $182.4 \pm 21.94$ and $203.3 \pm 34.76$ respectively. There was no statistical difference between the daily Gn dose used in the first $\mathrm{COH}$ and the daily $\mathrm{Gn}$ dose used in second $\mathrm{COH}$ $(p>0.05)$.

\section{Outcome for 24 cases with resuming COH-IVF-ET cycle}

Resuming the $\mathrm{COH}$ cycle was cancelled for seven patients without IVM when follicles showed no growth again around 13 $\mathrm{mm}$ with declined E2; 17 cases with resumed $\mathrm{COH}$ reached egg retrieval, though in one case no oocyte was retrieved. 16 cases produced embryos grading between I-III (according to the Racowsky scoring system in 2009), ${ }^{3}$ of which 14 cases underwent embryo transfer with day-3 embryos and two cases underwent embryo cryopreservation due to uterine cavity effusion. Twelve cases with embryo transfer resulted in clinical pregnancy (confirmed with intrauterine gestational sac by ultrasound 28-32 days after embryo transfer); two cases with embryo transfer resulted in no pregnancy (see Table 3). In Table 3 it shows that mean BMI for 17 patients with mature follicle and seven patients with ceased follicle growth in the second $\mathrm{COH}$ are $21.12 \pm 3.57$ and $29.29 \pm 3.20$, respectively. There is a statistically significant difference in mean BMI between the two groups of patient $(p<0.05)$

\section{Discussion}

PCOS is a common gynaecological end ocrine disorder that affects approximately $5-10 \%$ of women worldwide. Clinical characteristics include oligoovulation or anovulation, excess androgen and polycystic ovaries. It is one of the most common causes of infertility in reproductive age women. ${ }^{4-6}$ IVF-ET is a common treatment for PCOS patients with tubal factor, male factor, failed after multi-drugs for ovary stimulation and refractory PCOS patients. During IVF-ET treatment there are frequent cancellations due to several reasons which include abnormal follicle development due to resistance to stimulating drugs ${ }^{7}$ or ceased follicle growth; ${ }^{8}$ endometrium not synchronised with follicle growth; and ovarian hyperstimulation syndrome (OHSS) occurring during $\mathrm{COH}$ or after egg retrieval. ${ }^{9}$ At present, the long protocol with gonadotropin releasing hormone agonist ( $\mathrm{GnRHa}$ )/FSH or HMG/HCG is the traditional treatment for PCOS patients in IVF. However, the success rate is low for PCOS patients especially for patients with high BMI because of physiopathologically complexity, such as long-term chronic anovulation resulting in polycystic ovaries

\begin{tabular}{|c|c|c|c|}
\hline & Total Gn per case & $\begin{array}{l}\text { Days of } \\
\text { stimulations }\end{array}$ & $\begin{array}{l}\text { Daily Gn per } \\
\text { case }^{a}\end{array}$ \\
\hline First $\mathrm{COH}$ & $2,004 \pm 393.5$ & $11.0 \pm 1.79$ & $182.4 \pm 21.94$ \\
\hline $\begin{array}{l}\text { Second } \\
\mathrm{COH}\end{array}$ & $2,628 \pm 970.7$ & $12.8 \pm 3.10$ & $203.3 \pm 34.76$ \\
\hline
\end{tabular}

$\mathrm{COH}=$ controlled ovarian hyperstimulation; $\mathrm{Gn}=$ gonadotropin $\mathrm{a}=$ the daily $\mathrm{Gn}$ dose per case in first $\mathrm{COH}$ vs the daily $\mathrm{Gn}$ dose per case in second $\mathrm{COH}, \mathrm{p}>0.05$.

\begin{tabular}{|c|c|c|c|}
\hline & $\begin{array}{l}\text { Success to } \\
\text { mature follicle }\end{array}$ & $\begin{array}{l}\text { Fail to mature } \\
\text { follicle }\end{array}$ & $p$ value \\
\hline Cases, n & 17 & 7 & na \\
\hline BMI, $\mathrm{kg} / \mathrm{m}^{2}$ & $21.12 \pm 3.57$ & $29.29 \pm 3.20$ & 0.021 \\
\hline $\begin{array}{l}\text { Clinical } \\
\text { pregnancy, } n\end{array}$ & $12 / 14$ & na & na \\
\hline
\end{tabular}


and thickening ovarian cortex or not being sensitive to ovary stimulating drugs. Furthermore because the dose of HMG/FSH for follicle development is close to the threshold of OHSS, ${ }^{10,11}$ $\mathrm{COH}$ treatment is easily induced in OHSS in patients with PCOS. ${ }^{12}$ In recent years, IVM has been used to treat infertility caused by PCOS, and some progress has been made, but there are some problems such as low oocyte maturation rate, low fertilisation rate and low pregnancy rate. ${ }^{13}$ Traditionally, the 24 PCOS patients in this article experiencing ceased follicle growth around 10-14 $\mathrm{mm}$ with significantly declined $\mathrm{LH}$ and E2 during $\mathrm{COH}$ treatment would have been cancelled and restarted on the $\mathrm{GnRHa} / \mathrm{COH}$ cycle after two to three menstrual cycles. ${ }^{14,15}$ We observed their ovaries and endometrium after 15-20 days of discontinuing medication. The level of FSH/LH/ E2 and B ultrasounds all showed that patients were still under downregulation at the early follicle phase. We considered that the follicle was discharged or atresic after being insensitive to $\mathrm{Gn}$ during mid-late stage of first $\mathrm{COH}$, and high levels of E2 production in $\mathrm{COH}$ might cause negative feedback inhibition of the hypothalamic-pituitary-gonadal-axis, causing the ovary to be under downregulation at the early follicle phase. According to what is described above, we believed that PCOS patients would be under a long period of suppression after downregulating treatment. With approval from the ethical committee, we gave the 24 patients $G n$ in doses of 150-225 IU daily for second round of $\mathrm{COH}$. Seventeen patients developed mature follicles reaching oocyte retrieval, 16 of which had good quality of embryos. There were 14 patients with day-3 embryo transfers which resulted in good outcomes, with 12 clinical pregnancies. The remaining seven cases encountered the same problem of ceased follicle growth around $13 \mathrm{~mm}$. The mean E2 level of 17 cases in the second $\mathrm{COH}$ on the day of HCG trigger was less than 15,000 $\mathrm{pmol} / \mathrm{L}$, even though the daily dose of Gn was numerically higher than in their first $\mathrm{COH}$. In addition, no patients developed OHSS after their embryo transfers. More than half (17/24) of the patients developed mature follicles in the second $\mathrm{COH}$ with no statistical difference in doses of $\mathrm{Gn}$ in both $\mathrm{COHs}$, which explains why the ovaries, after downregulating for 40 days, were more sensitive to stimulating medication. The mean BMI of the seven patients with ceased follicle development again was higher than the others, which means that the drug sensitivity of the ovary was negatively correlated with BMI. Complete weight control should be an important way to increase the sensitivity to drugs, and can obtain the ideal clinical pregnancy outcome. The result shows that resuming $\mathrm{COH}$ treatment for patients with PCOS as described above is a feasible and safe option. This method has not been reported in domestic or foreign articles. It provides a new path for the treatment of PCOS, though it needs a larger sample of patients to draw a further conclusion. Another factor to consider is that in the traditional long protocol of treatment, $\mathrm{COH}$ will be utilised after 10-14 days of downregulation. This article shows that the downregulation status of PCOS patients lasts longer than usual in IVF patients. The question is whether or not we should start $\mathrm{COH}$ after 1 month of downregulation for PCOS patients.

\section{Conflicts of interest}

This work was supported by the National Natural Science Foundation of China (grant no 81571613) and funding provided by Sailing Fund of Fujian Medical University (QH038).
DONGHONG LI

Assistant researcher, The First Affiliated Hospital of Fujian Medical University, Fuzhou, China

YUJIA GUO

Professor, The First Affiliated Hospital of Fujian Medical University,

Fuzhou, China

SHUZHUANG LU

Professor, The First Affiliated Hospital of Fujian Medical University,

Fuzhou, China

YAO ZHONG

Professor, Reproductive Center for Women and Children Hospital, Changsha, China

WENBING ZHU

Professor, Reproductive Center for Women and Children Hospital, Changsha, China

QICAI LIU

Professor, The First Affiliated Hospital of Fujian Medical University,

Fuzhou, China

\section{References}

1 The Rotterdam ESHRE/ASRM-sponsored PCOS consensus workshop group. Revised 2003 consensus on diagnostic criteria and long-term health risks related to polycystic ovary syndrome. Hum Reprod 2004,19:41-7.

2 Haggerty CL, Ness RB. Diagnosis and treatment of pelvic inflammatory disease. Womens Health (Lond) 2008;4:383-97.

3 Kostov I, Ingilizova G. In vitro maturation - a potential approach of infertility treatment of patients with PCOS. Akush Ginekol (Sofiia) 2013;52:25-8.

4 Goodarzi MO, Dumesic DA, Chazenbalk G, Azziz R. Polycystic ovary syndrome: etiology, pathogenesis and diagnosis. Nat Rev Endocrinol 2011;7:219-31.

5 Lebbi I, Ben Temime R, Fadhlaoui A, Feki A. Ovarian drilling in PCOS: is it really useful. Front Surg 2015;17:30.

6 Zhao C, Liu X, Shi Z et al. Role of serum miRNAs in the prediction of ovarian hyperstimulation syndrome in polycystic ovarian syndrome patients. Cell Physiol Biochem 2015;35:1086-94.

7 Gong F, Tang Y, Zhang H, Lu G. Modified super-long downregulation protocol improved the outcome of in vitro fertilizationembryo transfer in infertile patients. Zhong Nan Da Xue Xue Bao Yi Xue Ban 2009;34:185-9.

8 Rose BI, Laky D. A comparison of the Cook single lumen immature ovum IVM needle to the Steiner-Tan pseudo double lumen flushing needle for oocyte retrieval for IVM. J Assist Reprod Genet 2013;30:855-60.

9 González-Ortega C, Piña-Aguilar RE, Cancino-Villarreal P, PérezPeña E, Gutiérrez-Gutiérrez AM. Natural-cycle in vitro fertilization (IVF) combined with in vitro maturation in infertile patients with polycystic ovarian syndrome (PCOS) requiring IVF. Taiwan J Obstet Gynecol 2019;58:192-5.

$10 \mathrm{Xu}$ Q, Chen J, Wei Z et al. Sex hormone metabolism and threatened abortion. Med Sci Monit 2017;23:5041-8.

11 Yu Q, Hu S, Wang Y et al. Letrozole versus laparoscopic ovarian drilling in clomiphene citrate-resistant women with polycystic ovary syndrome: a systematic review and meta-analysis of randomized controlled trials. Reprod Biol Endocrinol 2019;17:17.

12 Salmassi A, Mettler L, Hedderich ] et al. Cut-off levels of antimullerian hormone for the prediction of ovarian response, in vitro fertilization outcome and ovarian hyperstimulation syndrome. Int J Fertil Steril 2015;9:157-67. 
13 Xu YP, Xiang HF, Zou WW et al. Clinical application of in vitro maturation of human immature oocytes for infertile women with polycystic ovary syndrome. Zhonghua Fu Chan Ke Za Zhi 2012;47:14-8

14 Olivennes F, Belaisch-Allart J, Emperaire JC et al. Prospective, randomized, controlled study of in vitro fertilization embryo transfer with a single dose of a luteinizing hormone-releasing hormone (LH-RH) antagonist (cetrorelix) or a depot formula of an LH-RH agonist (triptorelin). Fertil Steril 2000;73:314-20.
15 Kamath MS, R R, Bhave P, K M, TK A, George K. Effectiveness of $\mathrm{GnRH}$ antagonist in intrauterine insemination cycles. Eur J Obstet Gynecol Reprod Biol 2013;166:168-72.

Address for correspondence: Dr Donghong Li and Prof Yujia Guo, The First Affiliated Hospital of Fujian Medical University, Fuzhou, Fujian 350001, China.

Email: donghong3721@126.com and guoyujia51@163.com 
The most useful

learning resource for

all MRCP(UK) exams

and beyond. A must-

Medical Masterclass

have for all physicians!

\section{Third edition textbooks now available}

\section{Choose the package or subscription that's best for you.}

\section{Printed textbooks}

The 12 printed textbooks cover the scientific background to medicine; common clinical presentations; planning a strategy for investigation and treatment; and the medical specialties that are covered in the MRCP(UK) exams.

The third edition of Medical Masterclass, is full of new content to aid your learning.

\section{Website subscriptions}

Buy the best website subscription for you, depending on the date of your exam.

Monthly subscriptions are available, and you can choose between access to MRCP(UK) Part 1 and Part 2 exam questions.

\section{The premier package}

The complete package gives you the full set of 12 printed textbooks and 1 year of unlimited access to the Medical Masterclass website.
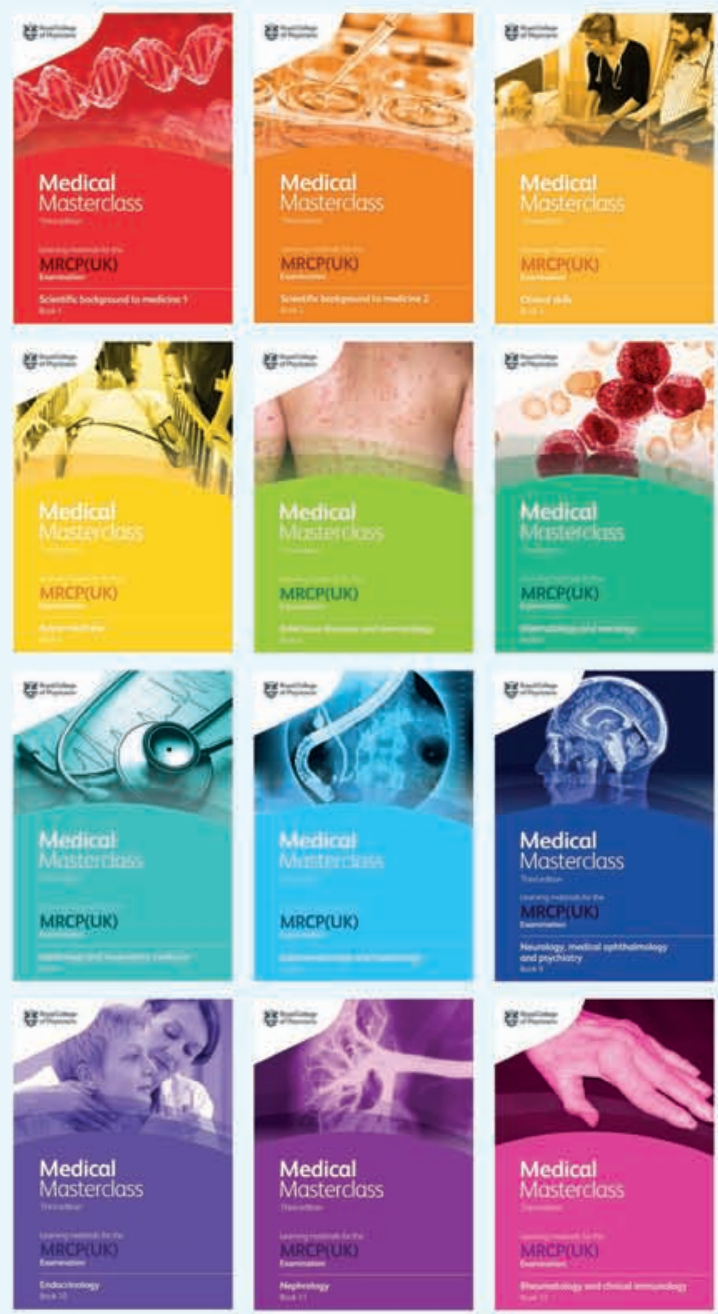